\title{
Bistable equilibrium points of mercury body burden.
}

\author{
Houman Owhadi*and Areen Boulos ${ }^{\dagger}$ \\ February 5, 2008
}

\begin{abstract}
In the last century mercury levels in the global environment have tripled as a result of increased pollution from industrial, occupational, medicinal and domestic uses [1]. Glutathione is known to be the main agent responsible for the excretion of mercury (we refer to [41], 38] and [35]). It has also been shown that mercury inhibits glutathione synthetase (an enzyme acting in the synthesization of Glutathione), therefore leading to decreased glutathione levels (we refer to [41], 34], [11] and [13]). Mercury also interferes with the production of heme in the porphyrin pathway [27]. Heme is needed for biological energy production and ability to detox organic toxins via the P450 enzymes [19]. The purpose of this paper is to show that body's response to mercury exposure is hysteretic, i.e. when this feedback of mercury on its main detoxifying agents is strong enough then mercury body burden has two points of equilibrium: one with normal abilities to detoxify and low levels of mercury and one with inhibited abilities to detoxify and high levels of mercury. Furthermore, a small increase of body's mercury burden may not be sufficient to trigger observable neurotoxic effects but it may be sufficient to act as a switch leading to an accumulation of mercury in the body through environmental exposure until its toxicity becomes manifest.
\end{abstract}

\section{The model}

Writing $C$ the mercury body burden a simple model for the evolution of $C$ with respect to time can be described by the following ordinary differential equation.

$$
\frac{d C}{d t}=\mu-k(C) C
$$

\footnotetext{
${ }^{*}$ California Institute of Technology Applied \& Computational Mathematics, Control \& Dynamical systems, MC 217-50 Pasadena, CA 91125, owhadi@caltech.edu

†areen@caltech.edu
} 
In equation (1), $\mu$ stands for the rate of exposure of the body to mercury. $k(C) C$ stands for the detoxification rate. As an instance of chronic exposure, recall that the intra oral air concentration in mercury is of the order of $2 \mu \mathrm{g} / \mathrm{m}^{3}$ without dental amalgam and $20 \mu \mathrm{g} / \mathrm{m}^{3}$ with dental amalgams [33. It has also been shown that dental amalgam filings in pregnant rats can be a prime source of exposure of fetal tissues to mercury [46].

Although equation (1) is a toy model we believe that it captures the main mechanism involved in mercury exposure. This toy model is based on the following assumptions:

- The excretion rate of mercury is proportional to the mercury body burden and we write $k(C)$ the proportionality constant.

- The proportionality constant $k(C)$ is a decreasing function of the mercury burden $C$ modeling the inhibition of organs ability to detoxify by mercury.

The model introduced in this paper is oversimplified but we believe that the associated switch mechanism could be of some relevance in the analysis of mercury toxicity.

Observe that one has to distinguish two main factors in mercury toxicity. The first one (addressed in this paper) is the total amount of mercury accumulated in the body. The second one (not addressed in this paper), is the mercury retention effects, i.e. the toxicity of a given value of mercury body burden (see [20], [10], [9] and the references therein).

- Low levels of mercury lead to aberrant porphyrin profile, the major product of the porphyrin pathway is heme and it is needed for biological energy production and ability to detoxify organic toxins via the P450 enzymes.

- Genetic susceptibilities that account for susceptibility to heavy metal toxicities (such as polymorphism in the CPOX4 or APO-E genes) are an important factor affecting excretion rates.

- Genetic variations of Apolipoprotein E (APO-E) ([19], 7], [18]) don't play a major role in mercury excretion but play a role in mercury retention effects by altering the detoxification abilities of the Central Nervous System by impacting the percentage of the total mercury body burden trapped in the cerebrospinal system. Hence although being an APO-E4 carrier won't significantly impact excretion rates it increases the susceptibility Alzheimer's disease ([19], [18]) by increasing the relative percentage of mercury withheld in the central nervous system. 
This being said, it is important to observe that although genetic variations may alter excretion rates $(k(C)$ would in practice depend on genetic susceptibilities), it would not explain a feed-back mechanism. Mercury is primarily excreted bound to glutathione ([41, 38] and [35]) and mercury can inhibit the production of glutathione [13] ([41], [34], [11] and [13]). We will show that through this feedback mechanism, very small doses of mercury cause a major breakdown of the detoxification pathway.

To simplify the presentation we will assume that $k(C)$ has the following form

$$
k(C)=k_{0} \max \left(1-\frac{C}{C_{0}}, \beta\right)
$$

where $k_{0}$ is the detoxification rate at low levels of mercury and $\beta$ is a constant between 0 and 1 indicating the decrease of the proportionality constant $k(C)$ under levels of mercury beyond $C_{0}(1-\beta)$. Thus $C_{0}$ is a positive parameter and $C_{0}(1-$ $\beta$ ) is a constant indicating a critical level of mercury beyond which Glutathione production is minimal. Figure $1(\mathrm{a})$ shows how $k(C)$ would vary as a function of $C$ in this model.

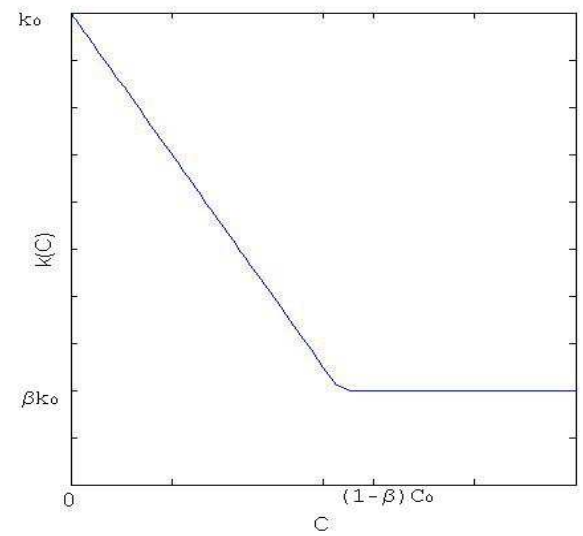

(a) $k(C)$ versus $C$.

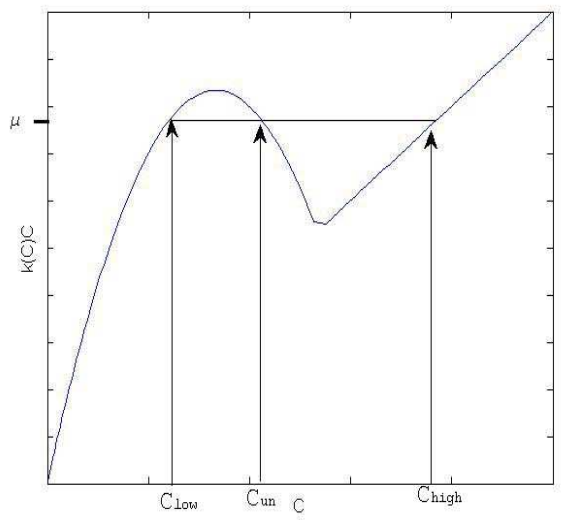

(b) $k(C) C$ versus $C$.

Figure 1: $k(C)$ and $k(C) C$ versus $C$.

One should observe that the phenomenon described here is independent of the particular choice of $k(C)$ and is solely based on the non monotonicity of $k(C) C$ with respect to $C$ as in figure $1(\mathrm{~b})$.

The equilibrium points for body mercury burden are solutions of the following equation

$$
k(C) C=\mu
$$


More precisely

$$
\max \left(1-\frac{C}{C_{0}}, \beta\right) \frac{C}{C_{0}}=\frac{\mu}{k_{0} C_{0}}
$$

Equation (41) has three solutions if an only if

$$
\beta(1-\beta)<\frac{\mu}{k_{0} C_{0}}<\frac{1}{4}
$$

Let us write $C_{\text {low }}<C_{\text {unstable }}<C_{\text {high }}$ those equilibrium points. Thus the dynamic of the body mercury burden can be divided into two classes.

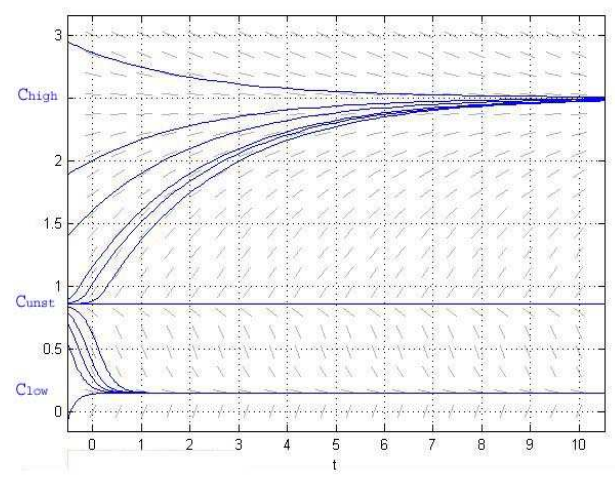

(a) Direction fields of mercury body burden.

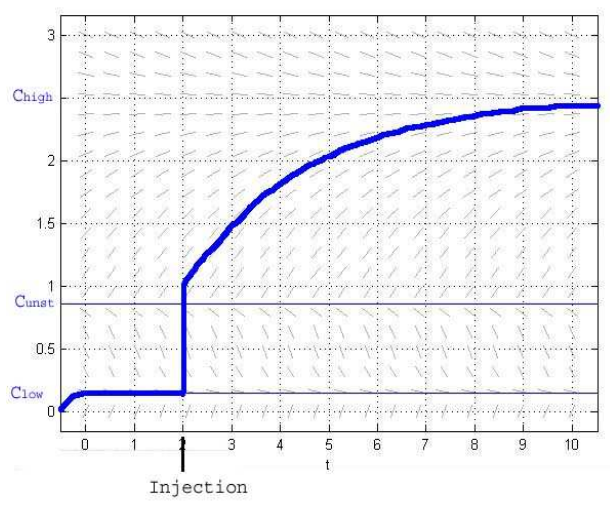

(b) Body burden is initially 0 with a low rate of exposure then at time 2 the body burden is increased in one moment.

Figure 2: Mercury burden dynamic.

Unique equilibrium point. This class is characterized by the fact that the chronic exposure to mercury $\mu$ is above the value $\frac{k_{0} C_{0}}{4}$ or below the value $\beta(1-$ $\beta) k_{0} C_{0}$. In this dynamic $C(t)$ will converge to the unique solution of (4) as $t \rightarrow \infty$.

Bistable equilibrium points. This class is characterized by the fact that $\beta<\frac{1}{2}$ (the decrease of $k(C)$ under high levels of mercury is at least $50 \%$ ) and that the chronic exposure to mercury $\mu$ is below the value $\frac{k_{0} C_{0}}{4}$ and above the value $\beta(1-\beta) k_{0} C_{0}$. In this case if $C(0) \in\left(0, C_{\text {unstable }}\right)$ then as $t \rightarrow \infty, C(t)$ will converge to $C_{\text {low }}$. If $C(0)>C_{\text {unstable }}$ then $C(t)$ will converge to $C_{\text {high }}$ as $t \rightarrow \infty$. $C_{\text {unstable }}$ is an unstable equilibrium point. We refer to figure 2(a). Observe also that in this 
situation a high mercury body burden can be induced by exposing the organism to a given small amount of mercury in a short lapse of time (see figure 2(b)].

Experimental detoxification rates. It would be interesting to obtain constant rates that apply to elemental $\mathrm{Hg}$, inorganic $\mathrm{Hg}$, and organic types of $\mathrm{Hg}$. While [43] contains excretion rate those rates needs to be put into correspondence with total body burden to infer the experimental estimation of the curve $k(C) C$. Such experimental data would be very valuable since it would allow us to compute the value $C_{\text {unstable }}-C_{\text {low }}$ (which may strain dependent) which is the amount of mercury leading to an activation of the switch mechanism if the body is exposed to it in one time.

Observe also that one could elaborate more sophisticated models where different organs have different detoxification rate (depending as well upon the chemical form of the mercury). Such models would put into evidence the biphasic blood detoxification rates for methyl mercury (with average half periods of 7-8 hours and 52 days). It is important to observe that the mechanism involved in the latter biphasic behavior (trapping of methyl-mercury by organs which are slow to detoxify) is different from one involved in this paper (inhibition of the ability to detoxify).

\section{Interpretation and predictions.}

According to the model presented in this paper, a transition from the low levels of mercury body burden to the high levels of mercury body burden can be induced by increasing the mercury body burden in a brief period of time (increasing $C$ through in a brief period of time) or increasing the rate of exposure $(\mu)$ over a long enough period of time. It is important to observe that to reach high levels of mercury body burden $C_{\text {high }}$ it is enough to increase the body burden by an amount $C_{\text {unstable }}-C_{\text {low }}$ which can be much smaller than $C_{\text {high }}-C_{\text {low }}$ (see figure 2(b)).

Observe that a prediction of our model is that once the metabolism is in the basin of attraction of inhibited abilities to detoxify it will start accumulating not only mercury, but also other heavy metals. Furthermore to induce a transition from inhibited abilities to detoxify to the basin of attraction of normal abilities to detoxify one would have to reduce the body mercury burden by an amount $C_{\text {high }}-C_{\text {unstable. }}$ It is important to observe that a transition from the low levels of mercury equilibrium point to the high levels of mercury equilibrium point can be induced by increasing the body mercury burden by an amount much smaller than levels corresponding to the difference between high levels and low levels of mercury burden but to reverse the process one would have lower body's mercury burden by a large amount. 


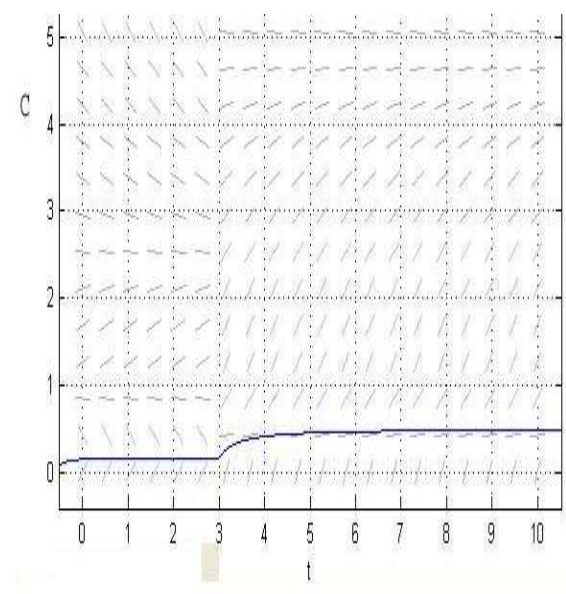

(a) Mercury body burden.

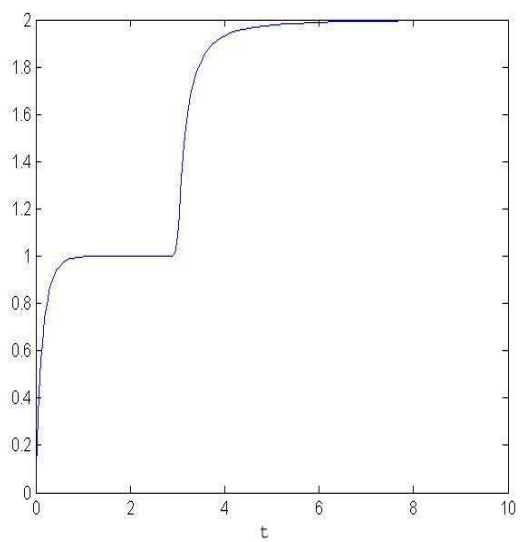

(b) Excretion rate.

Figure 3: At time 3 the rate of exposure is increased by $\Delta \mu<\Delta \mu_{c}$.

\section{Impact of an increase of the exposure rate $\mu$.}

In this section the assumptions behind the model are the following: at time zero the body burden is assumed to be zero, and the rate of exposure is assumed fixed. Then time three the rate of exposure $\mu$ is increased by a given amount $\Delta \mu$. We observe that if $\Delta \mu$ is smaller than a critical value $\Delta \mu_{c}$ (figure 3) then the effect effect is a small increase of the total mercury body burden (figure $3(\mathrm{a})$ ) and a corresponding increase of the rate of excretion of mercury (figure 3(b)). The mercury body burden remains on the equilibrium point associated to low levels of mercury and normal abilities to detoxify.

If $\Delta \mu$ is larger than the critical value $\Delta \mu_{c}$ (figure 4 then the effect is a large accumulation of mercury in the body (figure 4(a)). The rate of excretion (figure 4(b) starts increasing due the increase of mercury exposure, it reaches a maximum value then starts decreasing due to inhibited abilities to detoxify. Mercury accumulates in the organs until the equilibrium points corresponding to a high mercury burden is reached then the excretion rate increases again to match the exposure rate. In this second scenario the body burden bifurcates from low levels of mercury and normal abilities to detoxify to high levels of mercury and inhibited abilities to detoxify.

This theoretical observation based on our toy model is to be put in relation with s recent study [12] showing that after placing amalgams on children the urinary excretion of mercury starts increasing, reaches a peak at year 2, then drop over $40 \%$ in the next 5 years to the point where the error bars on the amalgam bearers 


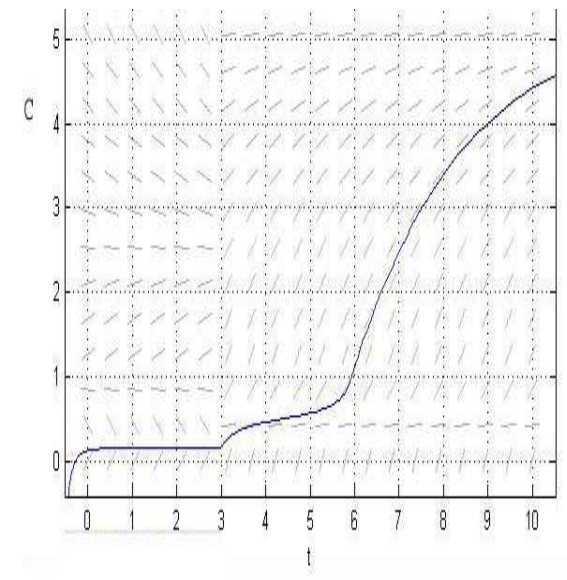

(a) Mercury body burden.

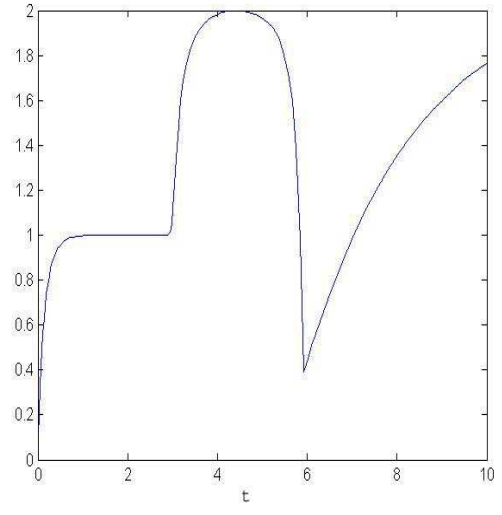

(b) Excretion rate.

Figure 4: At time 3 the rate of exposure is increased by $\Delta \mu>\Delta \mu_{c}$.

vs controls overlap. This drop in mercury excretion has been interpreted by the authors of [12] as proof of safety of dental amalgams. An other interpretation put forward in [20] is that children when exposed to mercury vapor for extended periods of time, slowly lose the ability to excrete mercury in their urine. Figure 4 show that an increase of the rate of exposure switch off the ability to detoxify and lead to a drop in the excretion rate after an initial period of increase.

\section{Mercury, a toxic time bomb [1].}

Mercury may be involved in several neurological disorders, where high mercury exposure at one moment or continuously could result in a reduced ability to detoxify (reduced $k(C)$ ).

First observe that toxic and neurotoxic effects of heavy metals [26] and in particular mercury [29] are well known. We refer to [6] for a review of human exposure to mercury and to [42] for an instance of acute exposure (Minamata disease).

Growth delay, reduced locomotion, exaggerated response to novelty, and densely packed, hyperchromic hippocampal neurons with altered glutamate receptors and transporters can be induced in mice following thimerosal challenges that mimic routine childhood immunizations [23. Thimerosal (ethylmercury) has been used as a preservative in vaccines since the 1930s [31. It has been shown in infant monkeys that [4] Thimerosal crosses the blood-brain barrier and transforms into inorganic mercury in the central nervous system. For strong evidences of the link 
between autism and mercury we refer to [17], [37] and [39].

Reduced levels of mercury have been found in first baby haircuts of autistic children indicating their inability to excrete mercury [22]. It has been observed in mice [23] that the sensitivity to thimerosal is strain dependent, this may indicate that detoxification abilities in the absence of mercury are already low in those strains or that the barrier $C_{\text {unstable }}-C_{\text {low }}$ is small enough in those strains to be crossed through injections of low amounts of ethylmercury.

We refer to [40] and 2] for the psychometric evidence that dental amalgam mercury may be an etiological factor in schizophrenia. In cerebrospinal fluid of drug-free schizophrenic patients, a significant decrease in the level of total glutathione has been observed as compared to controls [14].

We refer to [5] and [45] for the association between body burden mercury level and Parkinson's disease.

There is a significantly greater proportion of males than females affected by schizophrenia [36], Parkinson's disease [16] or autism [34], [11]. This has to be compared to the fact that at low levels of mercury, testosterone is known to enhance the neurotoxicity of mercury whereas oestrogen has protective properties [44].

It has been observed that blood mercury levels were more than two-fold higher in Alzheimer's disease patients as compared to control groups [21] (we also refer to [15]). It is also known [28] that seven of the characteristic markers of Alzheimer's disease can be produced in normal brain tissues, or cultures of neurons, by the addition of extremely low levels of mercury.

It is interesting to observe in that context that chelation therapies have been proposed for Parkinson's disease [8], Alzheimer's disease [30] and autism [24]. Furthermore high levels of mercury have been detected in autistic children following chelation therapy with DMSA [3], 25]. Furthermore urine excretion of mercury after DMPS challenge has been shown to be proportional to whole body burden before chelation [32].

A support of the detoxification pathway without the use of DMSA or DMPS has also been observed to lead a very significant increase of the rate of excretion of mercury (and other heavy metals) [47]. A strong link between detoxification and recovery from autism has been observed [24], [47].

Acknowledments. We would like to thank Boyd E. Haley for comments which have led to significant changes to this paper, for bringing to our attention the other factors at play in mercury toxicity besides Glutathione levels and indicating us references [12] and [20]. We would also like to thank two anonymous referees for valuable and detailed comments and suggestions. 


\section{References}

[1] Mercury exposure: The world's toxic time bomb. Technical report, Ban Mercury Working group, 2003. For the UNEP Governing Council Meeting February 3-7, Nairobi. Available at http://www.ban.org/Ban-Hg-Wg/.

[2] BADER A. Schizophrenia \& mercury poisoning. contribution to the study of the schizophreniforme psychoses due to mercury. Schweiz Arch Neurol Psychiatr., 68(2):209-241, 1952.

[3] Mark F. Blaxill, Lyn Redwood, and Sallie Bernard. Thimerosal and autism? a plausible hypothesis that should not be dismissed. Medical Hypotheses, 62:788794, 2004.

[4] Thomas M. Burbacher, Danny D. Shen, Noelle Liberato, Kimberly S. Grant, Elsa Cernichiari, and Thomas Clarkson. Comparison of blood and brain mercury levels in infant monkeys exposed to methylmercury or vaccines containing thimerosal. Environmental Health Perspectives, 113(8), 2005.

[5] Ngim CH and Devathasan G. Epidemiologic study on the association between body burden mercury level and idiopathic parkinson's disease. Neuroepidemiology, 8(3):128-141, 1989.

[6] Thomas W. Clarkson. The three modern faces of mercury. Environmental Health Perspectives, 110(1):11-23, 2002.

[7] EH Corder, AM Saunders, WJ Strittmatter, DE Schmechel, PC Gaskell, GW Small, AD Roses, JL Haines, , and MA Pericak-Vance. Gene dose of apolipoprotein e type 4 allele and the risk of alzheimer's disease in late onset families. Science.

[8] Ben-Shachar D, Eshel G, Riederer P, and Youdim MB. Role of iron and iron chelation in dopaminergic-induced neurodegeneration: implication for parkinson's disease. Ann Neurol., 32, 1992.

[9] Echeverria D, Woods JS, Heyer NJ, Rohlman D, Farin FM, and Garabedian CE Li T. The association between a genetic polymorphism of coproporphyrinogen oxidase, dental mercury exposure and neurobehavioral response in humans. Neurotoxicol Teratol.

[10] Echeverria D, Woods JS, Heyer NJ, Rohlman DS, Farin FM, Bittner AC Jr, and Garabedian C. Li T. Chronic low-level mercury exposure, bdnf polymorphism, and associations with cognitive and motor function. Neurotoxicol Teratol. 
[11] Geier DA and Geier MR. A clinical and laboratory evaluation of methionine cycle-transsulfuration and androgen pathway markers in children with autistic disorders. Horm Res, 66:33-39, 2006.

[12] Timothy A. DeRouen, Michael D. Martin, Brian G. Leroux, Brenda D. Townes, James S. Woods, Jorge Leitao, Alexandre Castro-Caldas, Henrique Luis, Mario Bernardo, Gail Rosenbaum, and Isabel P. Martins. Neurobehavioral effects of dental amalgam in children a randomized clinical trial. Journal of the American Medical Association.

[13] Richard C. Deth. Molecular aspects of thimerosal-induced autism. Technical report, Testimony Before the Subcommittee on Human Rights and Wellness, Committee on Government Reform, U.S. House of Representatives, 2004. Available at http://reform.house.gov/UploadedFiles/Testimony

[14] K. Q. Do, A. H. Trabesinger, M. Kirsten-Krger, C. J. Lauer, U. Dydak, D. Hell, F. Holsboer, P. Boesiger, and M. Cu 'enod. Schizophrenia: glutathione deficit in cerebrospinal fluid and prefrontal cortex in vivo. European Journal of Neuroscience, 12(10):3721, 2000.

[15] J. T. A. Ely. Mercury induced alzheimer's disease: Accelerating incidence? Bulletin of Environmental Contamination and Toxicology, 67(6):800-806, 2001.

[16] V E Bovbjerg J K Lee G F Wooten, L J Currie and J Patrie. Are men at greater risk for parkinsons disease than women? Journal of Neurology Neurosurgery and Psychiatry, 75:637-639, 2004.

[17] Robert Gunier Lisa A. Croen Gayle C. Windham, Lixia Zhang and Judith K. Grether. Autism spectrum disorders in relation to distribution of hazardous air pollutants in the san francisco bay area. Environ Health Perspect.

[18] Michael E. Godfrey, Damian P. Wojcik, and Cheryl A. Krone. apo-e genotyping. Journal of Alzheimer's Disease.

[19] Boyd E. Haley. 2006. Private communication.

[20] Boyd E. Haley. Response to the nidcr funded children's amalgam testing publications in the jama 2006. 2006. available at http://www.iaomt.org/documents/CAT_Haley_response.pdf.

[21] C. Hock, G. Drasch, S. Golombowski, F. Mller-Spahn, B. WillershausenZnnchen, P. Schwarz, U. Hock, J. H. Growdon, and R. M. Nitsch. Increased blood mercury levels in patients with alzheimer's disease. Journal of Neural Transmission, 105(1):59-68, 1998. 
[22] Amy S. Holmes, Mark F. Blaxill, and Boyd E. Haley. Reduced levels of mercury in first baby haircuts of autistic children. International Journal of Toxicology, 22(4):277 - 285, 2003.

[23] M Hornig, D Chian, and W I Lipkin. Neurotoxic effects of postnatal thimerosal are mouse strain dependent. Molecular Psychiatry, 9:833-845, 2004.

[24] AUTISM RESEARCH INSTITUTE. Treatment options for mercury/metal toxicity in autism and related developmental disabilities: Consensus position paper. 2005. available at http://www.autismwebsite.com/ARI/vaccine/heavymetals.pdf.

[25] Bradstreet J, Geier DA, Kartzinel JJ, Adams JB, and Geier MR. A. Casecontrol study of mercury burden in children with autistic spectrum disorders. J Am Phys Surg, 8:7679, 2003.

[26] Lars Järup. Hazards of heavy metal contamination. British Medical Bulletin, 68:167-182, 2003.

[27] Woods JS, Martin MD, Naleway CA, and Echeverria D. Urinary porphyrin profiles as a biomarker of mercury exposure: studies on dentists with occupational exposure to mercury vapor. J Toxicol Environ Health.

[28] Christopher C. W. Leong, Naweed I. Syed, and Fritz L. Lorscheider. Retrograde degeneration of neurite membrane structural integrity of nerve growth cones following in vitro exposure to mercury. Neuroreport, 12(4):733-737, 2001.

[29] Chang LW. Neurotoxic effects of mercury-a review. Environ Res., 14:329-373, 1977.

[30] KYLE Y. FAGÉT MATH P. CUAJUNGCO and, XUDONG HUANG, RUDOLPH E. TANZI, and ASHLEY I. BUSH. Metal chelation as a potential therapy for alzheimer's disease. Annals of the New York Academy of Sciences, 920:292, 2000.

[31] Pichichero ME, Cernichiari E, Lopreiato J, and Treanor J. Mercury concentrations and metabolism in infants receiving vaccines containing thiomersal: a descriptive study. Lancet, 360(9347):1737-1741, 2002.

[32] TW Clarkson MG Cherian, EF Miles and C Cox. Estimation of mercury burdens in rats by chelation with dimercaptopropane sulfonate. Pharmacology and Experimental Therapeutics, 245(2):479-484, 1988. 
[33] Vimy MJ and Lorscheider FL. Intra-oral air mercury released from dental amalgam. J Dent Res., 64:1069-1071, 1985.

[34] Geier MR and Geier DA. The potential importance of steroids in the treatment of autistic spectrum disorders and other disorders involving mercury toxicity. Med Hypotheses, 64:946-954, 2005.

[35] Lyn Patrick. Mercury toxicity and antioxidants: part i: role of glutathione and alpha-lipoic acid in the treatment of mercury toxicity - mercury toxicity. Toxicol Appl Pharmacol., 7:456-471, 2002.

[36] Lewine R, Burbach D, and Meltzer HY. Effect of diagnostic criteria on the ratio of male to female schizophrenic patients. Am J Psychiatry.

[37] Zachary Stein David Mandell Claudia Miller Raymond F. Palmera, Steven Blanchard. Environmental mercury release, special education rates, and autism disorder: an ecological study of texas. Health \& Place, 12:203209, 2006.

[38] Zalups RK, Barfuss DW, and Lash LH. Disposition of inorganic mercury following biliary obstruction and chemically induced glutathione depletion: dispositional changes one hour after the intravenous administration of mercuric chloride. Toxicol Appl Pharmacol., 154:135-144, 1999.

[39] Lorene Amet Alain Lam Anthea Springbett Richard Lathe Robert Nataf, Corinne Skorupka. Porphyrinuria in childhood autistic disorder: Implications for environmental toxicity. Toxicol. Appl. Pharmacol., 214(2):99108, 2006.

[40] RL SIBLERUD, J MOTL, and E KIENHOLZ. Psychometric evidence that dental amalgam mercury may be an etiological factor in schizophrenia. JOURNAL OF ORTHOMOLECULAR MEDICINE, 74, 1999.

[41] James SJ, Slikker W 3rd, Melnyk S, New E, Pogribna M, and Jernigan S. Thimerosal neurotoxicity is associated with glutathione depletion: protection with glutathione precursors. Neurotoxicology, 26:1-8, 2005.

[42] H. Matsumoto T. Takeuchi, N. Morikawa and Y. Shiraishi. A pathological study of minamata disease in japan. Acta Neuropathologica, 2(1):40-57, 1962.

[43] Dimiter L. Tsalev. Atomic Absorption Spectrometry in Occupational and Environmental Health Practice., pages 158-169. CRC Press, 1983. vol. 1.

[44] Clarkson TW, Nordberg GF, and Sager PR. Reproductive and developmental toxicity of metals. Scand J Work Environ Health, 11(3):145-154, 1985. 
[45] Finkelstein Y, Vardi J, Kesten MM, and Hod I. The enigma of parkinsonism in chronic borderline mercury intoxication, resolved by challenge with penicillamine. Neurotoxicology, 17(1):291-295, 1996.

[46] Takahashi Y, Tsuruta S, Hasegawa J, Kameyama Y, and Yoshida M. Release of mercury from dental amalgam fillings in pregnant rats and distribution of mercury in maternal and fetal tissues. Toxicology, 163:115-126, 2001.

[47] Amy Yasko and Dr. Garry Gordon. The Puzzle of Autism: Putting It All Together. Matrix Development Publishing, 2006. 2ND edition. 